and stable knock down of STAT3. Treatment of cardiomyocytes with shERK resulted in ERK1/2 phosphorylation and a decrease in the expression of CAR accompanied by the elevation of LDH levels in infected with $\mathrm{CVB}_{3}$ cells.

Conclusions In this study we investigated the effect of signalling through the Raf-MEK-ERK1/2 pathway on CAR expression in cardiac myocytes that are potential targets for adenovirus-based therapies. Our findings in modulation of CAR expression, may lead to new strategies in the gene therapy of DCM.

\title{
GW23-e2414 THE CROSSTALK BETWEEN ERK1/2 AND STAT3 IN THE REGULATION OF CAR EXPRESSION DURING CVB3 INFECTION IN CARDIOMYOCYTE.
}

doi:10.1136/heartjnl-2012-302920a.55

Jing-jin Liu, Bo Yu, Shuo Zhang. Harbin Medical University

Objectives We performed a novel analysis of cardiac expression of receptors for several adenovirus serotypes with a focus on expression of CAR, as adenoviruses targeting receptor has been used in various applications, that more than the viral receptor which is significantly induced in the heart tissue of $\mathrm{CVB}_{3}$ infected and considered to be the dominant aetiology of viral myocarditis.

Methods We treated cardiomyocytes with culture medium in the uninfected or infected of $\mathrm{CVB}_{3}(\mathrm{MOI}=100)$. The lentiviral vector system derived from HIV-1 was used to express short hairpin RNAs (shRNA) directed against $\mathrm{STAT}_{3}$ and ERK1/2 activation was blocked with U0126, an ERK1/2 inhibitor. Western blot was used to observe the level of CAR, ERK and $\mathrm{STAT}_{3}$. The degrees of cells injury were judged by LDH levels in cells supernatant.

Results Up-regulation activities of ERK1/2 after $\mathrm{CVB}_{3}$ infected with cardiomyocytes, accompanied by positive correlation of the expression of CAR. Treatment of cardiomyocytes with Pharmacological inhibition of ERK1/2 phosphorylation with U0126 resulted in a dramatic increase in the expression of CAR. U0126 induced the JNK/STAT 3 pathway activity to prevent cells from injury. Lentivector-based short hairpin RNAs provide efficient 